\title{
MOLECULAR BASIS OF INHERITED COLORECTAL CARCINOMAS IN THE MACEDONIAN POPULATION: AN UPDATE
}

\author{
Staninova-Stojovska $\mathrm{M}^{1}$, Matevska-Geskovska $\mathrm{N}^{1}$, Panovski $\mathrm{M}^{2}$, Angelovska B ${ }^{3}$, \\ Mitrevski $\mathrm{N}^{3}$, Ristevski $\mathrm{M}^{3}$, Jovanovic $\mathrm{R}^{4}$, Dimovski AJ $\mathrm{J}^{1,5}$,* \\ *Corresponding Author: Professor Aleksandar J. Dimovski, M.D., Ph.D., Center for Biomolecular \\ Pharmaceutical Analyses, UKIM Faculty of Pharmacy, Majka Tereza 47, and Research Center for Ge- \\ netic Engineering and Biotechnology, Macedonian Academy of Sciences and Arts, Krste Misirkov 2, \\ 1000 Skopje, RN Macedonia. Tel/Fax: +389-2-3235411. E-mail: adimovski@ff.ukim.edu.mk
}

\begin{abstract}
Hereditary factors are assumed to play a role in $\sim 35.0$ $45.0 \%$ of all colorectal cancers (CRCs) with about 5.0$10.0 \%$ associated with high penetrant disease-causing mutations in genes correlated to hereditary polyposis (HP) or hereditary non polyposis syndromes (HNPCC). Although inherited germline mutations in mismatch repair (MMR) and the $A P C$ genes contribute significantly to $\mathrm{CRC}$, genetic diagnosis cannot yet be obtained in more than $50.0 \%$ of familial cases. We present updated data of 107 probands from the Macedonian population with clinically diagnosed HP $(n=41)$ or HNPCC $(n=66)$ obtained by next generation sequencing (NGS) with three different gene panels covering the coding, flanking and promoter regions of 114 cancer predisposition genes. Using this approach, we were able to detect deleterious mutations in $65 / 107$ (60.7\%) patients, $50.4 \%$ of which were in known well-established CRC susceptibility genes and $10.2 \%$ in DNA repair genes (DRG). As expected, the highest frequencies of deleterious variants were detected in familial adenomatous polyposis (FAP) and in HNPCC patients with microsatellite instabil\footnotetext{
of Pharmacy, University "Ss. Cyril and Methodius", Skopje, RN Macedonia

${ }^{2}$ University Clinic for Abdominal Surgery, UKIM Faculty of Medicine, University "Ss. Cyril and Methodius", Skopje, RN Macedonia

${ }^{3}$ University Clinic for Radiotherapy and Oncology, UKIM Faculty of Medicine, University "Ss. Cyril and Methodius", Skopje, RN Macedonia

${ }^{4}$ Institute for Pathology, UKIM Faculty of Medicine, University "Ss. Cyril and Methodius", Skopje, RN Macedonia

${ }^{5}$ Research Center for Genetic Engineering and Biotechnology "Georgi D. Efremov", Macedonian Academy of Sciences and Arts, Skopje, RN Macedonia
}

${ }^{1}$ Center for Biomolecular Pharmaceutical Analyses, UKIM Faculty
\end{abstract}

ity (MSI) tumors (93.8 and 87.1\%, respectively). Variants of unknown significance (VUS) were detected in 24/107 (22.4\%) patients, mainly in HNPCC patients with microsatellite stable (MSS) tumors or patients with oligopolyposis. The majority of VUS were also found in DRG genes, indicating the potential role of a doble-strand brake DNA repair pathway deficiency in colorectal cancerogenesis. We could not detect any variant in $18 / 107(16.8 \%)$ patients, which supports the genetic heterogeneity of hereditary CRC, particularly in HNPCC families with MSS tumors and in families with oligopolyposis.

Keywords: Hereditary colorectal cancer (CRC); Macedonian population; mutations.

\section{INTRODUCTION}

Colorectal cancer (CRC) is one of the most frequent cancers, especially in well-developed countries and it is one of the major public health concerns worldwide. Hereditary factors are assumed to play a role in approximately 35.0$45.0 \%$ of all CRCs [1]. Our understanding of the genetic basis, as well as the guidelines for clinical management of hereditary CRC syndromes continue to evolve rapidly, so it is crucial for clinicians to recognize the unique features in the diagnosis and management of these syndromes. A precise understanding of the genetics of inherited CRCs is important for identifying at-risk individuals, improving cancer surveillance and prevention strategies and developing better diagnostic and therapeutic approaches [2,3].

Approximately $5.0-10.0 \%$ of hereditary CRCs develop due to highly penetrant mutations in genes associated with well-characterized inherited Mendelian cancer syndromes. The most commonly affected genes are the MMR genes [MLH1 (OMIM: 120436); MSH2 (OMIM: 609309); MSH6 
(OMIM: 600678) and PMS2 (OMIM: 600259)] in patients with the Lynch syndrome, and the $A P C$ gene (OMIM: 611731 ) in patients with familial adenomatous polyposis (FAP). Current data indicate that the molecular defects in these syndromes are very heterogeneous, and that certain mutations might occur with considerable frequencies in particular populations [3]. In the remaining $30.0 \%$ of inherited CRCs, the etiology is still not completely understood. The recent expansion of new sequencing technologies based on massive parallel next generation sequencing (NGS), including whole-genome and whole-exome sequencing, as well as copy number approaches, offered opportunities for identification of new high-penetrant genes that could explain the aggregation of CRC in high-risk families [4]. New insights in this field open considerable challenges concerning variant interpretations even for the known CRC susceptibility genes. Next generation sequencing data provide a huge number of variants in both coding and non coding regions; thus, identifying a disease-causing variant from this large number of variants poses a serious task.

Another possible explanation for the aggregation of $\mathrm{CRCs}$ in certain families is that the heritability is not due to a single monogenic defect but a multifactorial condition, caused by the conjunction of moderate-risk or low-risk genetic variants, possibly in combination with environmental or lifestyle risk factors. Evidence from recent studies shows that the accumulation of risk variants is associated significantly with an increased risk of CRC in individuals with a family history of the disease [5].

We have previously reported our initial data on the molecular characterization of FAP and HNPCC in our population $[6,7]$. We now present updated results of the study, which was performed on a larger cohort of patients with a clinical diagnosis of hereditary CRCs using an extended panel of genes related to cancer predispositions.

\section{MATERIALS AND METHODS}

A total of 107 probands included in this study were recruited from the University Clinics for Digestive Surgery and for Radiology and Oncology, Skopje, RN Macedonia. Sixty- six patients comply with Amsterdam criteria for clinical diagnosis of HNPCC and 41 patients were diagnosed with multiple polyps reminiscent of FAP $(>1000$ adenomatous polyps through the large bowel, attenuated FAP ( $<100$ adenomatous polyps) or juvenile polyposis (multiple polyps with hemartomatous component). Informed consent was obtained from all participants. The research protocol was approved by the Ethics Committee of the Faculty of Pharmacy in Skopje, RN Macedonia.

Whole blood ( $3 \mathrm{~mL}$ with EDTA as anticoagulant) and fresh frozen tumor tissue or formalin-fixed paraffinembed- ded (FFPE) blocks were used for DNA isolation. DNA was extracted using the standard phenol/chloroform method and quantified using the spectrophotometer NanoDrop 2000 (Thermo Fisher Scientific, Waltham, MA, USA). Microsatellite instability in tumor samples was analyzed with a multiplex fluorescent polymerase chain reaction (PCR), followed by capillary electrophoresis (CE) on a 3500 Automated Genetic Analyzer (Life Technologies, Carlsbad, CA, USA). Microsatellite instability (MSI) status was determined using nine short tandem repeat (STR) markers: BAT25, BAT26, D2S123, D5S107, D5S346, D17S250, D18S58, D18S61 and D18S535, derived from the panel of microsatellite loci defined by the National Cancer Institute and the protocols described previously $[7,8]$. The tumor samples were classified as MSI-H (MSIhigh) if instability was present at more than $30.0 \%$ of the loci screened, MSI-L (MSI-low) if at least one but fewer than $30.0 \%$ of the loci showed instability or MSS (microsatellite stable) if all loci were stable.

Four deferent multiplex ligation-dependent probe amplification (MLPA) analyses were used for screening for extended germline rearrangements in the $M L H 1$, MSH2, MSH6, PMS2 and APC genes. The HNPCC samples were ligated and amplified using the SALSA MLPA P003 (MLH1/MSH2) SALSA MLPA P072 (MSHO) and SALSA MLPA P008 (PMS2) probe mixes according to the manufacturer's recommendations [Microbiology Research Centre Holland (MRC-Holland), Amsterdam, The Netherlands]. In patients manifesting oligopolyposis, the whole $A P C$ gene (exon 1-18) and a substantial part of the MUTYH gene (exons 1, 4 and 9) were screened for large deletions/duplications using the SALSA MLPA P043 $(A P C)$ probemix. Data normalization and analysis for both analyses were conducted using Coffalyser.Net software (MRC-Holland; http://www.mlpa.com).

The presence of methylation in the $M L H 1$ gene promoter was analyzed in DNA isolated from tumors of all patients with MSI positive non polyposis familial CRC. For this analysis, $1 \mu \mathrm{g}$ of DNA was converted by bisulfite modification using EZ DNA Methylation-Lightning Kit (Zymo Research, Irvine, CA, USA) following the procedure recommended by the manufacturer. A total of 50-100 ng of converted DNA was subjected to real-time PCR analysis using primers and fluorescent probes specific for methylated DNA in the $M L H 1$ gene promoter region [9]. The efficacy of the bisulfite modification was evaluated by a control reaction, which was run in parallel for every sample with primers and probe located in the $A C T B$ gene that does not contain $\mathrm{CpG}$ islands and is not subjected to methylation.

Two different platforms were used for NGS: Ion Torrent PGM ${ }^{\mathrm{TM}}$ (Thermo Fisher Scientific) and MiniSeq (Illumina Inc., San Diego, CA, USA). For the Ion Tor- 
rent PGM platform, two custom AmpliSeq panels were designed using AmpliSeq Designer (Life Technologies). First, analyses of the genes most commonly affected in HNPCC and FAP (MLH1, MSH2, MSH6 PMS2 and $A P C)$ were conducted and all negative samples were additionally analyzed using a second panel consisting of all genes implicated in HNPCC or familial polyposis (POLE, POLD1, MUTYH, SMAD4, BMPR1A, STK11, GREM1, PTEN, SEMA4A, RPS20, FAN1, POT1, MRE11A, LIMK2, $I L 12 R B 1)$. In both cases, DNA libraries were prepared using Ion AmpliSeq ${ }^{\mathrm{TM}}$ Library Kit 2.0 (Life Technologies), barcoded using Ion Xpress ${ }^{\mathrm{TM}}$ Barcode Adapters Kit (Thermo Fisher Scientific), and normalized with Library EqualizerTM Kit (Thermo Fisher Scientific), multiplexed according to the manufacturer's protocols, to an amount permitting a theoretical coverage of at least 100 reads per targeted sequence/patient. Template preparation and sample enrichment was conducted on Ion OneTouch ${ }^{\mathrm{TM}} 2$ System (Thermo Fisher Scientific) using Ion PGMTM Hi-Q OT2 Kit (Thermo Fisher Scientific). The sequencing reactions were carried on an Ion torrent PGM $^{\mathrm{TM}}$ platform using Ion PGM ${ }^{\mathrm{TM}} \mathrm{Hi}-\mathrm{Q}$ Sequencing Kit and Ion $316^{\mathrm{TM}}$ Chips v2 (Ion torrent; Life Technologies). The data were aligned using TMAP (Torrent Suite Software; Life Technologies) and annotated using the Variant caller (Torrent Suite Software; Thermo Fisher Scientific) and ANNOVAR (http://annovar. openbioinformatics.org) [10]. All patients in whom no causative mutations were found in the genes from the two custom panels, were analyzed using TruSight Cancer Panel (Illumina Inc.), which targets 94 genes and 284 SNPs associated with a predisposition to various cancers. DNA libraries were prepared and multiplexed using Trusight Rapid Capture kit, according to the manufacturer's instructions. The sequencing reactions were performed on a MiniSeq platform (Illumina Inc.) using MiniSeq Mid Output Kit (300-cycles). The data were aligned and annotated using the Variant Studio v.3 software (Illumina Inc.). The potential pathogenicity of the detected variants of unknown significance (VUS) was tested using the software for functional prediction [PolyPhen2 (http://genetics. bwh. harvard.edu.pph2/), SIFT (https://sift.bii.a-star.edu. sg/), FATHMM (http://fathmm.biocompute.org.uk), Mutation Taster (http://www.mutationtaster. org), PROVEAN (http://provean.jcvi.org/index.php), VARSOME (https:// varsome.com)] and for their clinical impact [ClinVar; (https://www.ncbi.nlm.nih.gov/clinvar) and InSight (https:// www.insight-group.org/variants/data bases/)]. For final classification of the detected sequence variants, we used the recommended guidelines from the American Collage of Medical Genetics (ACMG) [11]. The variants classified by this approach as pathogenic or likely pathogenic, are referred to as deleterious variants in the text.
All potentially deleterious variants and low coverage regions were validated using standard protocols for Sanger sequencing. In addition, the region spanning exons 10-15 of the PMS 2 gene were initially amplified by two long range PCR reaction spanning exons 10 to 12 and 12 to 15 , respectively, using primers that discriminate the $P M S 2$ gene from the highly homologous $P M S 2 C L$ pseudogene sequences. These PCR products were used as templates for the amplification of individual exons of the PMS2 gene. Prior to the sequencing reaction, the amplicons were purified using a low-melt agarose protocol [12]. Bidirectional sequencing was performed using BigDye Terminator v3.1 Cycle Sequencing Kit (Thermo Fisher Scientific). Sequencing products were purified using BigDye XTerminator ${ }^{\circledR}$ Purification Kit (Thermo Fisher Scientific) and analyzed with CE on a 3500 Genetic Analyzer (Thermo Fisher Scientific). The reference sequences used for variant nomenclature are given in Supplementary Table 1.

\section{RESULTS}

After a detailed review of the clinical/pathological data and familial segregation of CRC and other cancers, we selected 107 unrelated patients for molecular analysis, of which 66 probands complied with the Amsterdam criteria for clinical diagnosis of HNPCC and 41 had multiple polyps reminiscent of FAP or associated syndromes (Figure 1). Of the $66 \mathrm{HNPPC}$ patients, 31 had the MSI+phenotype and 35 had MSS tumors. The disease in HNPCC patients with MSI+ tumors developed predominantly in males, at a younger age (average 42.5 years, range $24-75$ years) and with a preponderance for the proximal colon, whereas in HNPCC patients with MSS tumors, the onset of the disease was at an average age of 53 years (range 17-81 years), equally distributed in both genders and localizations. Of the 41 patients with polyposis, 16 patients, predominantly

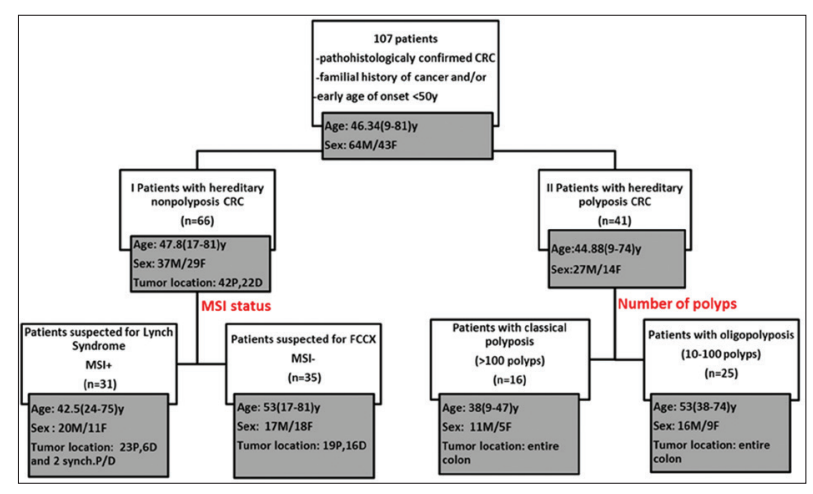

Figure 1. Number of patients in different subgroups based on the number of polyps and MSI status of their tumors at diagnosis. M: male; F: female; P: Proximal colon (caecum, ascendens, transversum); D: distal colon (descendens, sigma, rectum). 
males (11/16), presented with a classical FAP phenotype ( $>1000$ polyps) at an average age of 38 years (range 9-47 years) at diagnosis, while 25 patients with oligopolyposis $(<100$ polyps $)$ were diagnosed at an average age of 53 years (range 38-74 years). Extracolonic cancers were present in 17/31 families with MSI+ HNPCC (primarily endometrial and gastric cancers), in 23/35 families with MSS HNPCC (primarily breast/ovarian, endometrial and gastric cancers and leukemias); in 2/16 families with FAP (both with gastric cancer) and in 7/25 families with oligopolyposis (primarily breast and pancreatic cancers). The clinical and pathological data of these patients are summarized in Table 1.

Molecular Analysis of Patients with HNPCC. Overall, deleterious variants were detected in 37/66 (56.0\%) patients with HNPCC, of which 29/66 (44.0\%) in MMR and 8/66 (12.0\%) in DRG (DNA repair pathway) genes (Figure 2). The majority of patients with MSI+ tumors $(25 / 31 ; 80.6 \%)$ carried deleterious mutations in the MMR genes. Generally, we have identified seven different pathogenic variants in the $M L H 1$ gene in 15 unrelated patients, three different pathogenic variants in the $M L H 2$ gene in five patients, and three different pathogenic variants in the PMS2 gene in five patients (Table 1). It is worth noting that five variants were present in $>50.0 \%$ of the MSI+HNPCC patients [MLH1: c.392C $>$ G p.(Ser131Ter) in six families; MLH1: c. $244 \mathrm{~A}>\mathrm{G}$ p.(Thr82Ala), $M$ SH2: c.2211-2A $>\mathrm{C}$ p.(?) and PMS2: c.(803+1_804-1)_(*1_?)del p.(?) each in three families, and $M L H \overline{1}$ : c.896_897insC p.(Pro300Ser fsTer7) in two families]. Apart from these, all other identified variant were found in individual families; two missense mutations [c.62C $>$ T p. (Ala21 Val) and c.683T $>C$ p.(Leu228Pro)], one frameshift variant [c.1602del p.(Asn 535IlefsTer56)] and one splice variant [c.1667+1del p.(?)] in the $M L H 1$ gene, two in-frame deletions [c.1786 1788 del p.(Asn596del) and c.209 211+11del p.(?)] in the MSH2 gene and two small insertions/deletions [(c.2192 2196 del p.(Leu731Cys fsTer3) and c.1327del p.(Arg443 GlufsTer5)] in the PMS2 gene. The MSI+ phenotype in the remaining six patients from this group was due to the presence of $M L H 1$ promoter methylation (six patients) or bi-allelic somatic inactivation of the $\mathrm{MSH} 2$ gene (one patient). In one of these patients we detected germline VUS [c.418G $>$ A p.(Ser128 Leu)] in the PMS2 gene, in another two patients we found deleterious germline variants in the CHEK2 [c.470T $>$ G p.Ile157Ser ] and FANCL [c.2T $>$ C p.Met1Thr] genes, and one patient had a VUS in the FANCM gene [c.643G $>$ A p.(Glu215Lys)]. We did not detect any VUS/pathogenic anomalies in the 114 cancer predisposition genes tested in only two patients from the $\mathrm{MSI}+\mathrm{HNPCC}$ group. It is worth noting that the mutation c. $1799 \mathrm{~T}>\mathrm{A}$ p.Val600Glu in the $B R A F$ gene was present

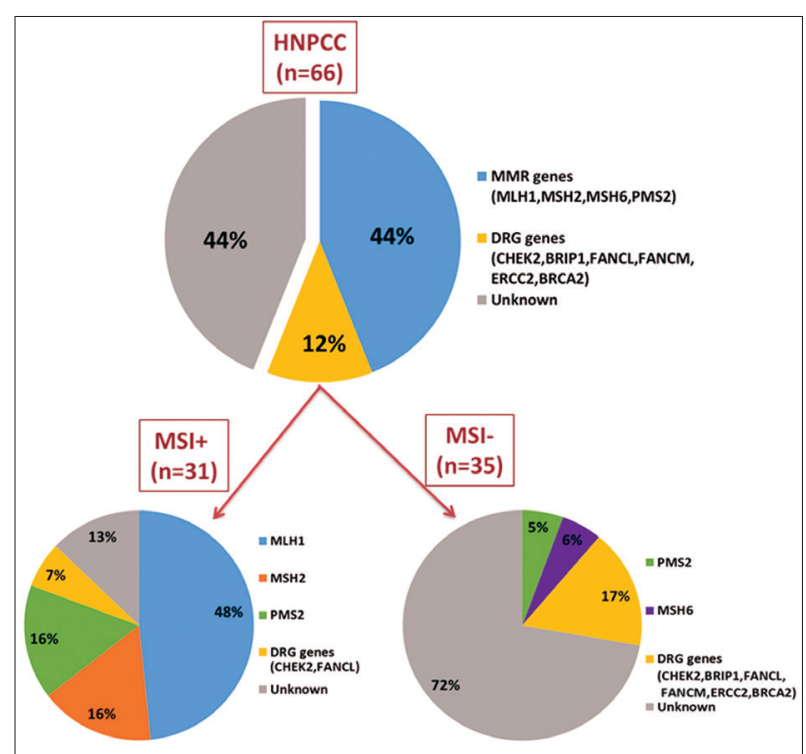

Figure 2. Distribution of deleterious variants in 66 patients with HNPCC. The distribution of the mutations in different genes in the two subgroups of HNPCC patients divided by the MSI status of their tumors is also shown.

only in the patient with the FANCL pathogenic variant from this subgroup. Overall, of the 14 pathogenic and two VUS detected in this group of patients, five and one variants, respectively are novel, and nine and one variants, respectively, have been reported previously and are present in the relevant databases (Table 1).

Deleterious variants were detected in 10/35 (28.0\%) HNPCC patients with MSS tumors (Figure 2 and Table 1), of which three variants in the MMR genes in four patients (11.4\%) [c.(?_-152)_(260+1_261-1) p(?) and c.457+1G $>$ T p.(?) in MSH6 and one gene inversion in the PMS2 gene in two patients] and five in five different DRG genes in six patients (17.0\%) [BRIP1: c.2392C $>\mathrm{T}$ p.(Arg798Ter) in two patients, BRCA2: c.4446_4451dupAACAGA, p.(Glu 1482 Thr1483dup), CHEK2: c.1100del p.Thr367fs FANCM: c.2953del p.(Glu985ArgfsTer3) and ERCC2: c. $1403 \mathrm{C}>\mathrm{T}$ p.(Pro468Leu), in one patient each]. A large percentage of patients from this group $(14 / 35,40.0 \%)$ had a VUS, of which four in known CRC genes (MMR and $A P C)$, eight in DRG genes (BLM, CHEK2, FANCL and $P A L B 2)$ and four in genes with different functions ( $E Z H 2$, CEP57, KIT, CDH1). The VUS c.545C $>$ T p.(Thr182Ile) in the $B L M$ gene was present in a patient with a $B R C A 2$ [c.4446_4451 dup AACAGA p.(Glu1482_Thr1483dup)] likely pathogenic variant, whereas two patients had two VUS, one of which was in the PALB2 gene [c.2792T $>\mathrm{G}$ p.(Leu $931 \mathrm{Arg})]$ and the second variant in the $A P C$ [c.4073C $>$ T p.(Ala1358Val) and KIT c.1688T $>$ A p.(Ile 563Lys)] genes, respectively. In $11 / 35$ (31.5\%) of the HNPCC patients with MSS tumors we did not detect any 
BALKAN JOURNAL OF MEDICAL GENETICS

Staninova-Stojovska M, Matevska-Geskovska N, Panovski M, Angelovska B,

Mitrevski N, Ristevski M, Jovanovic R, Dimovski AJ

Table 1. Summary of clinical data and molecular defects detected in 66 patients with hereditary nonpolyposis colorectal cancer.

\begin{tabular}{|c|c|c|c|c|c|c|c|c|c|c|c|c|}
\hline$n$ & \begin{tabular}{c|} 
Sex- \\
Age
\end{tabular} & $\begin{array}{c}\text { History } \\
\text { (relatives affected) }\end{array}$ & $\begin{array}{l}\text { Tumor } \\
\text { Location }\end{array}$ & $\begin{array}{l}\text { Stage } \\
\text { at } D_{x}\end{array}$ & $\begin{array}{c}\text { Extracolonic } \\
\text { Cancers }^{\mathrm{a}}\end{array}$ & Gene & $\begin{array}{l}\text { DNA Sequence } \\
\text { Change }\end{array}$ & $\begin{array}{l}\text { Amino Acid } \\
\text { Change }\end{array}$ & \begin{tabular}{|c|} 
ACMG \\
Classification \\
[11]
\end{tabular} & & & \\
\hline & & & & & & & & & & 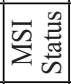 & 焉 & 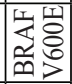 \\
\hline 1 & M-42 & $1: 1^{\text {st }} ; 2: 2^{\text {nd }}$ degree & transversum & na & G; P; Ur & MLH1 & c.896_897insC & p.Pro300SerfsTer7 & pathogenic & {$[+]$} & {$[-]$} & {$[-]$} \\
\hline 2 & M-55 & $3: 1^{\text {st }} ; 1: 2^{\text {nd }}$ degree & transversum & na & none & MLH1 & c. $392 \mathrm{C}>\mathrm{G}$ & p.Ser131Ter & pathogenic & {$[+]$} & {$[-]$} & {$[-]$} \\
\hline 3 & F-49 & $1: 1^{\text {st }} ; 1: 2^{\text {nd }}$ degree & transversum ${ }^{\mathrm{b}}$ & IIA & G; E & MLHI & c. $392 \mathrm{C}>\mathrm{G}$ & p.Ser131Ter & pathogenic & {$[+]$} & {$[-]$} & {$[-]$} \\
\hline 4 & M-32 & $1: 1^{\text {st }} ; 2: 2^{\text {nd }}$ degree & transversum & IIA & none & MLH1 & c. $392 \mathrm{C}>\mathrm{G}$ & p.Ser131Ter & pathogenic & {$[+]$} & {$[-]$} & {$[-]$} \\
\hline 5 & M-33 & $1: 1^{\text {st }} ; 2: 2^{\text {nd }}$ degree & ascendens & IIA & none & MLH1 & c. $392 \mathrm{C}>\mathrm{G}$ & p.Ser131Ter & pathogenic & {$[+]$} & {$[-]$} & {$[-]$} \\
\hline 6 & F-48 & $\begin{array}{l}\text { positive family } \\
\text { history; NS }\end{array}$ & ascendens & na & E & $M L H 1$ & c. 1602 del & p.Asn535IlefsTer56 & pathogenic & {$[+]$} & {$[-]$} & {$[-]$} \\
\hline 7 & F-43 & $2: 1^{\text {st }} ; 3: 2^{\text {nd }}$ degree & descendens & na & $\mathrm{G} ; \mathrm{P} ; \mathrm{Ur}$ & MLH1 & c.896_897insC & p.Thr372ThrfsTer7 & pathogenic & {$[+]$} & {$[-]$} & {$[-]$} \\
\hline 8 & F-60 & $2: 1^{\text {st }} ; 2: 2^{\text {nd }}$ degree & caecum & IIA & none & MLH1 & c. $392 \mathrm{C}>\mathrm{G}$ & p.Ser131Ter & pathogenic & {$[+]$} & {$[-]$} & {$[-]$} \\
\hline 9 & F-41 & $1: 1^{\text {st }} ; 2: 2^{\text {nd }}$ degree & caecum & na & E & MLH1 & c. $1667+1$ del & p.? & pathogenic & {$[+]$} & {$[-]$} & {$[-]$} \\
\hline 10 & F-29 & $\begin{array}{l}1: 1^{\text {st }} ; 2: 2^{\text {nd }} ; 2: 3^{\text {rd }} \\
\text { degree }\end{array}$ & descendens & na & none & MLH1 & c. $392 \mathrm{C}>\mathrm{G}$ & p.Ser131Ter & pathogenic & {$[+]$} & {$[-]$} & {$[-]$} \\
\hline 11 & F-24 & $1: 1^{\text {st }} ; 1: 2^{\text {nd }}$ degree & caecum & IIA & E & MLH1 & c. $244 \mathrm{~A}>\mathrm{G}$ & p.Thr82Ala & \begin{tabular}{|l} 
likely \\
pathogenic
\end{tabular} & {$[+]$} & {$[-]$} & {$[-]$} \\
\hline 12 & M-40 & 1: $1^{\text {st }}$ degree & ascendens & IIA & none & MLH1 & c. $244 \mathrm{~A}>\mathrm{G}$ & p.Thr82Ala & \begin{tabular}{|l|} 
likely \\
pathogenic
\end{tabular} & {$[+]$} & {$[-]$} & {$[-]$} \\
\hline 13 & M-55 & 1: $2^{\text {nd }}$ degree & caecum & IIA & none & MLH1 & c. $244 \mathrm{~A}>\mathrm{G}$ & p.Thr82Ala & $\begin{array}{l}\text { likely } \\
\text { pathogenic }\end{array}$ & {$[+]$} & {$[-]$} & {$[-]$} \\
\hline 14 & M-38 & $\begin{array}{l}\text { 2: } 1^{\text {st }} ; 2: 2^{\text {nd }} ; 2: 3^{\text {rd }} \\
\text { degree }\end{array}$ & ascendens & IIA & P; B & MLHI & c. $62 \mathrm{C}>\mathrm{T}$ & p.Ala21Val & $\begin{array}{l}\text { likely } \\
\text { pathogenic }\end{array}$ & {$[+]$} & {$[-]$} & {$[-]$} \\
\hline 15 & F-57 & $1: 1^{\text {st }} ; 2: 2^{\text {nd }}$ degree & ascendens & na & B & $M L H 1$ & c. $683 \mathrm{~T}>\mathrm{C}$ & p.Leu228Pro & \begin{tabular}{|l|} 
likely \\
pathogenic
\end{tabular} & {$[+]$} & {$[-]$} & {$[-]$} \\
\hline 16 & M-15 & $1: 1^{\text {st }} ; 2: 2^{\text {nd }}$ degree & transversum & IIA & E & MSH2 & c. $2211-2 \mathrm{~A}>\mathrm{C}$ & p.? & pathogenic & {$[+]$} & {$[-]$} & {$[-]$} \\
\hline 17 & F-41 & $2: 1^{\text {st }} ; 2: 2^{\text {nd }}$ degree & caecum & IIA & $\mathrm{E}$ & MSH2 & c. $2211-2 \mathrm{~A}>\mathrm{C}$ & p.? & pathogenic & {$[+]$} & {$[-]$} & {$[-]$} \\
\hline 18 & M-50 & $\begin{array}{l}\text { 1: } 1^{\text {st }} ; 2: 2^{\text {nd }} ; 2: 3^{\text {rd }} \\
\text { degree }\end{array}$ & transversum & IIA & E & MSH2 & c. $2211-2 \mathrm{~A}>\mathrm{C}$ & p.? & pathogenic & {$[+]$} & {$[-]$} & {$[-]$} \\
\hline 19 & M-41 & $3: 2^{\text {nd }}$ degree & rectosygma & IIIC & $\mathrm{E}$ & MSH2 & c.209_211+11del & p.? & pathogenic & {$[+]$} & {$[-]$} & {$[-]$} \\
\hline 20 & M-46 & $\begin{array}{l}\text { 1: } 1^{\text {st }} ; 4: 2^{\text {nd }} ; 2: 3^{\text {rd }} \\
\text { degree }\end{array}$ & rectum & na & G & MSH2 & c. $1786-1788 \mathrm{del}$ & p.Asn596del & \begin{tabular}{|l} 
likely \\
pathogenic
\end{tabular} & {$[+]$} & {$[-]$} & {$[-]$} \\
\hline 21 & F-31 & $1: 1^{\text {st }} ; 2: 2^{\text {nd }}$ degree & ascendens & IVA & none & MSH6 & \begin{tabular}{|l|} 
c. $(?-152)$ \\
$\left(26 \overline{0}+1 \_\overline{1} 1-1\right)$ \\
\end{tabular} & p.? & pathogenic & {$[-]$} & NA & {$[-]$} \\
\hline 22 & F-44 & $1: 1^{\text {st }} ; 2: 2^{\text {nd }}$ degree & rectum & IIIB & E & MSH6 & c. $458+1 \mathrm{G}>\mathrm{T}$ & p.? & pathogenic & {$[-]$} & NA & {$[-]$} \\
\hline 23 & F-44 & $2: 1^{\text {st }} ; 2: 2^{\text {nd }}$ degree & transversum & IIIA & $\mathrm{O}$ & MSH6 & c. $2384 \mathrm{~T}>\mathrm{C}$ & p.Ile795Thr & VUS & {$[-]$} & NA & {$[-]$} \\
\hline 24 & M-81 & 1: $1^{\text {st }}$ degree & rectum & IIIC & none & $P M S 2$ & \begin{tabular}{|l|} 
gene inversion \\
\end{tabular} & - & pathogenic & {$[-]$} & NA & {$[-]$} \\
\hline 25 & M-61 & 1: $1^{\text {st }}$ degree & sygma & IIIB & none & PMS2 & gene inversion & - & pathogenic & {$[-]$} & NA & {$[-]$} \\
\hline 26 & M-31 & 1: $1^{\text {st }}$ degree & caecum & IIA & none & PMS2 & $\begin{array}{l}\text { c. }\left(803+1 \_804-1\right) \\
\left(* 1 \_?\right) \text { de }{ }^{2}\end{array}$ & p.? & pathogenic & {$[+]$} & {$[-]$} & {$[-]$} \\
\hline 27 & M-39 & 1: $1^{\text {st }}$ degree & ascendens & IIIC & E & PMS2 & 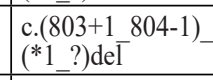 & p.? & pathogenic & {$[+]$} & {$[-]$} & {$[-]$} \\
\hline 28 & M-68 & $1: 1^{\text {st }}, 1: 2^{\text {nd }}$ degree & \begin{tabular}{|l|}
$\begin{array}{l}\text { rectosygma } \\
\text { caecum }\end{array}$ \\
\end{tabular} & IIA & none & PMS 2 & c.2192_2196del & p.Leu731CysfsTer3 & pathogenic & {$[+]$} & {$[-]$} & {$[-]$} \\
\hline 29 & M-65 & 1: $1^{\text {st }}$ degree & caecum & na & none & PMS2 & c.1327del & p.Pro443ThrfsTer16 & pathogenic & {$[+]$} & {$[+]$} & {$[-]$} \\
\hline 30 & M-40 & $\begin{array}{l}\text { positive family } \\
\text { history; NS }\end{array}$ & caecum & na & none & PMS2 & $\begin{array}{l}\text { c. }(803+1 \text { 804-1) } \\
(* 1 \text { *1_)de- }\end{array}$ & p.? & pathogenic & {$[+]$} & {$[-]$} & {$[-]$} \\
\hline 31 & $\mathrm{~F}-53$ & 2: $2^{\text {nd }}$ degree & $\begin{array}{l}\begin{array}{l}\text { caecum }+ \\
\text { rectum }\end{array} \\
\end{array}$ & IIIB & E & PMS2 & c. $418 \mathrm{G}>\mathrm{A}$ & p.Ser128Leu & VUS & {$[+]$} & {$[+]$} & {$[-]$} \\
\hline 32 & F-59 & $3: 1^{\text {st }} ; 1: 2^{\text {nd }}$ degree & rectum & IIA & E; G; T; L & $P M S 2$ & c. $934 \mathrm{~A}>\mathrm{G}$ & p.Met312Val & VUS & {$[-]$} & NA & {$[-]$} \\
\hline 33 & F-53 & $\begin{array}{l}2: 1^{\text {st }} ; 3: 2^{\text {ndd }} ; 3: 3^{\text {rd }} \\
\text { degree }\end{array}$ & sygma & in situ & $\mathrm{L}$ & PMS2 & c. $726 \mathrm{G}>\mathrm{A}$ & p.Gly207Glu & VUS & {$[-]$} & NA & {$[-]$} \\
\hline 34 & M-53 & 2: $1^{\text {st }}$ degree & caecum & IIIA & B & CHEK2 & c.1100del & p.Thr367fs & pathogenic & {$[-]$} & NA & {$[-]$} \\
\hline 35 & M-52 & $\begin{array}{l}\text { positive family } \\
\text { history; NS }\end{array}$ & caecum & na & none & CHEK2 & c. $470 \mathrm{~T}>\mathrm{G}$ & p.Ile157Ser & $\begin{array}{l}\text { likely } \\
\text { pathogenic }\end{array}$ & {$[+]$} & {$[+]$} & {$[-]$} \\
\hline
\end{tabular}


$>$ continuation from the previous page

\begin{tabular}{|c|c|c|c|c|c|c|c|c|c|c|c|c|}
\hline 36 & F-51 & $\begin{array}{l}\text { 1: } 1^{\text {st }} ; 1: 2^{\text {nd }} ; 2: 3^{\text {rd }} \\
\text { degree }\end{array}$ & rectum & na & $\mathrm{B} ; \mathrm{P}$ & CHEK2 & c. $374 \mathrm{~T}>\mathrm{G}$ & p.Phe125Cys & VUS & {$[-]$} & NA & {$[-]$} \\
\hline 37 & F-59 & $\begin{array}{l}\text { positive family } \\
\text { history; NS }\end{array}$ & sygma & IV & none & CHEK2 & c. $1313 \mathrm{~A}>\mathrm{G}$ & p.Asp438Gly & VUS & {$[-]$} & NA & {$[-]$} \\
\hline 38 & M-57 & $\begin{array}{l}\text { positive family } \\
\text { history; NS }\end{array}$ & ascendens & IIIB & none & FANCL & c. $2 \mathrm{~T}>\mathrm{C}$ & p.Met1Thr & pathogenic & {$[+]$} & {$[+]$} & {$[+]$} \\
\hline 39 & F-43 & $3: 2^{\text {nd }} ; 1: 3^{\text {rd }}$ degree & transversum & IIA & $\mathrm{G} ; \mathrm{R}$ & FANCL & $\begin{array}{l}\text { c.1111_1114dup } \\
\text { ATTA- }\end{array}$ & p.Thr372Asnfs & VUS & {$[-]$} & NA & {$[-]$} \\
\hline 40 & M-64 & 2: $1^{\text {st }}$ degree & rectum & IIIC & none & FANCL & $\begin{array}{l}\text { c.1111_1114dup } \\
\text { ATTA }\end{array}$ & p.Thr372Asnfs & VUS & {$[-]$} & NA & {$[-]$} \\
\hline 41 & $\mathrm{~F}-42$ & 1: $1^{\text {st }}$ degree & caecum & na & E; B & FANCM & c. $2953 \mathrm{del}$ & p.Glu985ArgfsTer3 & pathogenic & {$[-]$} & NA & {$[-]$} \\
\hline 42 & M-75 & 2: $1^{\text {st }} ; 1: 2^{\text {nd }}$ degree & rectum & na & G & FANCM & c. $643 \mathrm{G}>\mathrm{A}$ & p.Glu215Lys & VUS & {$[+]$} & NA & {$[-]$} \\
\hline 43 & F-53 & $1: 1^{\text {st }} ; 1: 3^{\text {rd }}$ degree & transversum & I & none & BRIP1 & c. $2392 \mathrm{C}>\mathrm{T}$ & p.Arg798Ter & pathogenic & {$[-]$} & NA & {$[-]$} \\
\hline 44 & M-55 & $\begin{array}{l}1: 1^{\text {st. }}, 1: 2^{\text {nd }} ; 2: 3^{\text {rd }} \\
\text { degree }\end{array}$ & ascendens & IIIC & $\mathrm{L}$ & BRIP1 & c. $2392 \mathrm{C}>\mathrm{T}$ & p.Arg798Ter & pathogenic & {$[-]$} & NA & {$[-]$} \\
\hline 45 & M-50 & $1: 1^{\text {st }}, 3: 2^{\text {nd }}$ degree & rectum & IIB & $\mathrm{E} ; \mathrm{G}$ & ERCC2 & c. $1403 \mathrm{C}>\mathrm{T}$ & p.Pro468Leu & pathogenic & {$[-]$} & NA & {$[-]$} \\
\hline 46 & F-38 & $1: 1^{\text {st }} ; 4: 2^{\text {nd }}$ degree & ascendens & na & G; P & $B L M$ & c. $481 \mathrm{G}>\mathrm{A}$ & p.Asp161Asn & VUS & {$[-]$} & NA & {$[-]$} \\
\hline 47 & M-60 & 2: $1^{\text {st }}$ degree & rectum & IIA & $\mathrm{O} ; \mathrm{P}$ & $\begin{array}{l}B R C A 2 ; \\
B L M\end{array}$ & $\begin{array}{l}\text { c. } 4446 \text { 4451dup; } \\
\text { c.545C>T } \\
\text { AACAGA }\end{array}$ & $\begin{array}{l}\text { p.Glu1482- } \\
\text { Thr1483dup; } \\
\text { p.Thr182Ile }\end{array}$ & $\begin{array}{l}\text { likely } \\
\text { pathogenic; } \\
\text { VUS }\end{array}$ & {$[-]$} & NA & {$[-]$} \\
\hline 48 & M-44 & 1: $1^{\text {st }} ; 2: 2^{\text {nd }}$ degree & transversum & IIIB & $\mathrm{T}$ & $\begin{array}{l}A P C \\
P A L B 2\end{array}$ & $\begin{array}{l}\text { c. } 4073 \mathrm{C}>\mathrm{T} ; \\
\text { c. } 2792 \mathrm{~T}>\mathrm{G}\end{array}$ & $\begin{array}{l}\text { p.Ala1358Val; } \\
\text { p.Leu931Arg }\end{array}$ & VUS; VUS & {$[-]$} & NA & {$[-]$} \\
\hline 49 & M-55 & $1: 1^{\text {st }} ; 1: 2^{\text {nd }}$ degree & caecum & IIIB & none & $\begin{array}{l}\text { KIT; } \\
\text { PALB2 }\end{array}$ & $\begin{array}{l}\text { c. } 1688 \mathrm{~T}>\mathrm{A} ; \\
\text { c. } 2792 \mathrm{~T}>\mathrm{G}\end{array}$ & $\begin{array}{l}\text { p.Ile563Lys; } \\
\text { p.Leu931Arg }\end{array}$ & VUS; VUS & {$[-]$} & NA & {$[-]$} \\
\hline 50 & F-70 & 2: $1^{\text {st }}$ degree & sygma & IIB & none & $\mathrm{CDHI}$ & c. $1348 \mathrm{~T}>\mathrm{A}$ & p.(Tyr450Asn) & VUS & {$[-]$} & NA & {$[-]$} \\
\hline 51 & M-59 & 1: $1^{\text {st }}$ degree & ascendens & na & none & CEP57 & c. $154 \mathrm{C}>\mathrm{T}$ & p.Arg52Cys & VUS & {$[-]$} & NA & {$[-]$} \\
\hline 52 & F-50 & $2: 1^{\text {st }} ; 1: 2^{\text {nd }}$ degree & transversum & na & B & EZH2 & c. $821 \mathrm{G}>\mathrm{A}$ & p.Arg274Lys & VUS & {$[-]$} & NA & {$[-]$} \\
\hline 53 & M-17 & $1: 2^{\text {nd }}$ degree & rectum & IIIC & $\mathrm{R}$ & KIT & c. $2484 \mathrm{C}>\mathrm{T}$ & p.Asn828Asn & VUS & {$[-]$} & NA & {$[-]$} \\
\hline 54 & M-49 & 2: $2^{\text {nd }}$ degree & caecum & na & E & unknown & {$[-]$} & {$[-]$} & {$[-]$} & {$[-]$} & NA & {$[-]$} \\
\hline 55 & M-37 & $\begin{array}{l}\text { positive family } \\
\text { history; NS } \\
\end{array}$ & caecum & IIB & none & unknown & {$[-]$} & {$[-]$} & {$[-]$} & {$[+]$} & {$[+]$} & {$[-]$} \\
\hline 56 & M-47 & $2: 1^{\text {st }} ; 1: 2^{\text {nd }}$ degree & caecum & IIA & none & unknown & {$[-]$} & {$[-]$} & {$[-]$} & {$[-]$} & NA & {$[-]$} \\
\hline 57 & M-67 & $\begin{array}{l}\text { 3: } 1^{\text {st. }}, 1: 2^{\text {nd }} ; 2: 3^{\text {rd }} \\
\text { degree }\end{array}$ & caecum & IIIB & $\mathrm{E} ; \mathrm{B}$ & unknown & {$[-]$} & {$[-]$} & {$[-]$} & {$[-]$} & NA & {$[-]$} \\
\hline 58 & F-43 & $1: 2^{\text {nd }}$ degree & transversum & na & none & unknown & {$[-]$} & {$[-]$} & {$[-]$} & {$[-]$} & NA & {$[-]$} \\
\hline 59 & F-30 & $1: 2^{\text {nd. }}: 1: 3^{\text {rd }}$ degree & rectum & IIIC & none & unknown & {$[-]$} & {$[-]$} & {$[-]$} & {$[-]$} & NA & {$[-]$} \\
\hline 60 & M-49 & $3: 2^{\text {nd }}$ degree & rectum & na & $\mathrm{Br}$ & unknown & {$[-]$} & {$[-]$} & {$[-]$} & {$[-]$} & NA & {$[-]$} \\
\hline 61 & F-64 & 2: $1^{\text {st }} ; 2: 2^{\text {nd }}$ degree & rectum & IIB & $\mathrm{L} ; \mathrm{B}$ & unknown & {$[-]$} & {$[-]$} & {$[-]$} & {$[-]$} & NA & {$[-]$} \\
\hline 62 & F-62 & $2: 1^{\text {st }} ; 1: 3^{\text {rd }}$ degree & crassl & na & E & unknown & {$[-]$} & {$[-]$} & {$[-]$} & {$[-]$} & NA & {$[-]$} \\
\hline 63 & M-65 & $1: 1^{\text {st. }} ; 3: 2^{\text {nd }}$ degree & rectosygma & IIIC & E & unknown & {$[-]$} & {$[-]$} & {$[-]$} & {$[-]$} & NA & {$[-]$} \\
\hline 64 & F-53 & $1: 1^{\text {st }}$ degree & crassl & IV & $\mathrm{B} ; \mathrm{E}$ & unknown & {$[-]$} & {$[-]$} & {$[-]$} & {$[-]$} & NA & {$[-]$} \\
\hline 65 & F-38 & 1: $1^{\text {st }}$ degree & caecum & IIA & E & unknown & {$[-]$} & {$[-]$} & {$[-]$} & {$[+]$} & {$[+]$} & {$[-]$} \\
\hline 66 & F-78 & 2: $1^{\text {st }}$ degree & ascendens $^{\mathrm{c}}$ & na & $\mathrm{R} ; \mathrm{B} ; \mathrm{Ur}$ & unknown & {$[-]$} & {$[-]$} & {$[-]$} & {$[-]$} & NA & {$[-]$} \\
\hline
\end{tabular}

$\mathrm{D}_{\mathrm{X}}$ : diagnosis; ACMG: classification according to the American Collage of Medical Genetics [11]; MSI: microsatellite instability; MLH1 met: methylation of the promoter of the MLH1 gene; BRAF V600E; V600E somatic mutation in the BRAF gene; VUS: variant of unknown significance; NS: not specified; NA: not analyzed.

a G: gastric cancer; P: prostate cancer; Ur: ureteral cancer; E: endometrial cancer; B: breast cancer; O: ovarian cancer; T: thyroid cancer; L: leukemia; R: renal cancer; Br: brain tumor: no mutation detected; [-]: absent; na: not available

b Synchronous: colon + gastric cancer, 5 years before endometrial cancer.

c Synchronous: colon + gastric cancer.

pathogenic or VUS in the 114 genes tested. The general clinical and pathohistological characteristics of these patients were similar to those of the other patients from the HNPCC MSS subgroup.
Molecular Analysis of Patients with Polyposis. On the whole, deleterious variants were detected in 28/41 (68.0\%) patients with polyposis (Figure 3, Table 2). In the FAP group, deleterious variants were detected in 15/16 
(93.7\%) patients, most of which $(13 / 16 ; 81.2 \%)$ were located in the $A P C$ gene. Two novel large deletions removing the entire $A P C$ gene were detected in four unrelated families [c. $-19+5016 * 2113+20168 d e l$ p.(?) $(123,466 \mathrm{bp}$ deletion) and c. $-19+2475 * 2113+34050$ del p.(?) $(139,889$ bp deletion), in two families each]. Four previously described small out-of-frame deletions in exon 15 [c.3927 $3931 \mathrm{del}$ p.(Glu1309AspfsTer4) and c.3183_3187del p.(Gln1062Terfs), found in two patients each, c.3404 3405del p.(Tyr1135fsTer) and c.3199_3202del p.(Ser1068 GlyfsTer57)] and three nonsense mutations [c.1269G $>$ A p.(Trp423Ter), c.904C $>$ T p.(Arg302Ter) and c.1660C $>$ T p.(Arg554Ter)] were also detected. In one patient with juvenile polyposis (presence of hemartomatous polyps), we detected a novel mutation in the 5' untranslated region (5'UTR) exon of the BMPR1A gene [c.-152-2A >G p.(?)]. Surprisingly, a pathogenic variant was found in the FLCN gene [c.1285insC: p.(His429ProfsTer27)] in one family with classical FAP presentation. A VUS in the KIT gene [c.2484C $>$ T p. $($ Asn $828=)$ ] was detected in one patient with deleterious mutation in the BMPR1A gene. Only one of the FAP patients did not have a pathogenic/VUS in any of the 114 genes tested.

The molecular defect in patients with oligopolyposis was extremely heterogeneous (Figure 3, Table 2). Deleterious variants were detected in $13 / 25(52.0 \%)$ of the patients. In 11/25 (44.0\%), the variants were located in genes associated with polyposis syndromes, including $A P C$ [c.256A > T p.(Lys86Ter) and c.3920T >A p.(Ile1307Lys)],

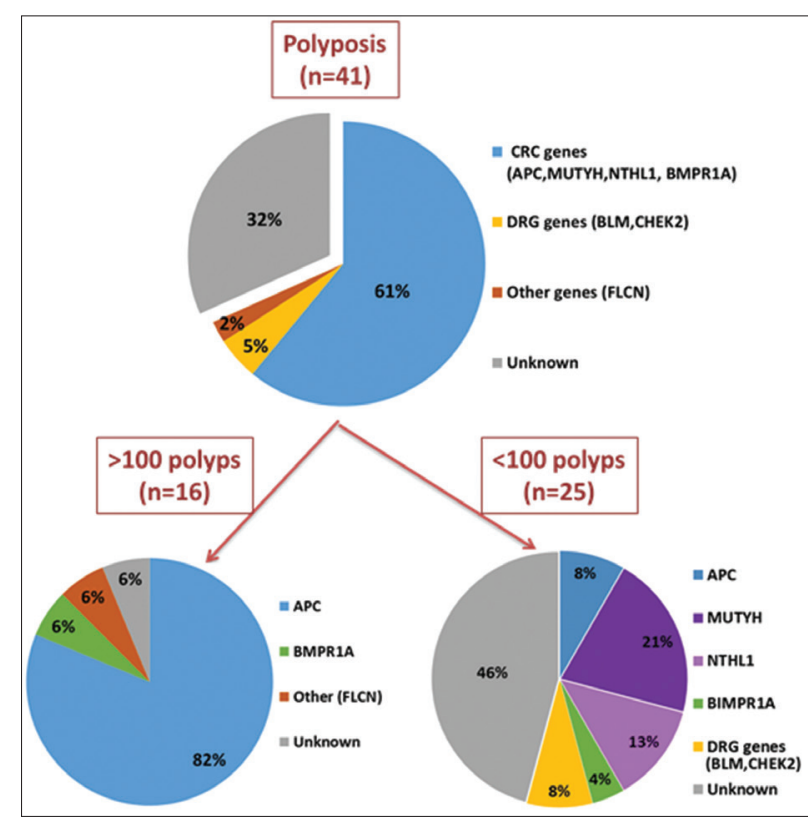

Figure 3. Distribution of deleterious variants in 41 patients with polyposis syndrome. The distribution of the mutations in different genes in the two subgroups of patients divided by the number of polyps at diagnosis is also shown.
MUTYH ([c.734G $>$ A]; [c.734G $>$ A] [p.(Arg245His)]; [p. $(\operatorname{Arg} 245 \mathrm{His})])$ in two patients and $([\mathrm{c} .536 \mathrm{~A}>\mathrm{G}]$; $[$ c. $536 \mathrm{~A}=][\mathrm{p} .($ Tyr179Cys $)] ;[\mathrm{p} .($ Tyr179=)] in three patients), NTHL1 ([c.268G >A]; [c.806C > T] [p.(Gln90 Ter)]; [p.(Trp269Ter)]) in one patient and [c.268G $>$ A]; [c.268=] [p. $(\mathrm{Gln} 90 \mathrm{Ter})] ;[\mathrm{p} .(\mathrm{Gln} 90=)]$ in two patients $])$ and $B M$ $P R 1 A[$ c.1A $>$ G p.(Met1Val)] in one patient) genes. The other three deleterious variants were detected in three different genes, all in the DRG pathway [BLM c.1642C $>\mathrm{T}$ p.(Gln548Ter), CHEK2 c.902del p.(Leu301TrpfsTer3) and FANCL c.2T $>$ C p.(Met1?)]. The FANCL mutation was detected in the patient that was heterozygous for the $M U$ TYH c.536A $>$ G p.(Tyr179Cys) mutation. A total of eight different VUS were detected in $8 / 25(32.0 \%)$ patients. Six of these variants were found as individual genetic changes, two in ATM [c.2149C $>$ T p.(Arg717Trp) and c.9016G $>$ C p.(Ala3006Pro)], one in PALB2 [c.1846G $>$ C p.(Asp616His)], one in MRE11 [c.1462C > T p.(Arg488 Cys) in two patients], one in POLE [c. 2527A $>$ G p.(Ile843 Val)] and in one $F H$ [c.1431_1433 dupAAA p.(Lys477 dup)] gene. One patient was detected with three VUS in different genes [BLM c.3416G $>$ C p.(Arg1139Pro), MRE11 c. $1462 \mathrm{C}>\mathrm{T}$ p.(Arg488Cys) and DIS3L2 c.1447 $\mathrm{C}>\mathrm{G}$ p.(Arg483 Gly)], while one novel VUS in the RUNX1 gene $[\mathrm{c} .711 \mathrm{G}>\mathrm{C}$ p.(Gln237His)] was found in the patient with a mono-allelic deleterious mutation in the NTHL1 gene. Only four patients from this group did not have any variant in the 114 genes tested. It is worth noting that extracolonic cancers (breast, pancreatic, endometrial) were present in family members of patients with mutations in NTHL1, CHEK2 and BLM genes, in 1/4 patients without any genetic variant (breast cancer) and were absent in family members of patients with VUS.

\section{DISCUSSION}

Using the approach described above, we were able to detect clear pathogenic variants (deleterious mutations) in 65/107 (60.7\%) patients with hereditary CRCs in our population, of which 54/107 (50.5\%) in known well-established CRC susceptibility genes and 11/107 (10.2\%) in other genes [Figure 4(A) and Figure 5(A)]. Similar results were obtained in several large studies of patients with a hereditary cancer using whole exome sequencing $[13,14]$, supporting the role of our approach for cascade testing of this disorder.

The majority of these deleterious variants were detected in HNPCC families with MSI+tumors and families expressing the FAP phenotype [Figure 4(B)]. In the HNPCC group, the Lynch syndrome was confirmed in 25/31 $(80.6 \%)$ of the MSI+ patients and in $4 / 35(11.4 \%)$ patients with MSS tumors, indicating the need for Lynch syndrome 
Table 2. Summary of clinical data and molecular defects detected in 41 patients with polyposis syndromes.

\begin{tabular}{|c|c|c|c|c|c|c|c|c|c|c|}
\hline$n$ & $\begin{array}{l}\text { Sex- } \\
\text { Age }\end{array}$ & Clinical $D_{x}$ & \begin{tabular}{|c|}
$\begin{array}{c}\text { Number } \\
\text { of } \\
\text { Polyps }\end{array}$ \\
\end{tabular} & \begin{tabular}{|c|}
$\begin{array}{c}\text { Type } \\
\text { of } \\
\text { Polyps }\end{array}$ \\
\end{tabular} & $\begin{array}{l}\text { Family History } \\
\text { (relatives affected) }\end{array}$ & $\begin{array}{c}\text { Extracolonic } \\
\text { Cancers }^{b}\end{array}$ & $\begin{array}{l}\text { Affected } \\
\text { Gene }\end{array}$ & DNA Sequence Change & Amino Acid Change & \begin{tabular}{|c|} 
ACMG \\
Classi- \\
fication [11]
\end{tabular} \\
\hline 1 & M-40 & FAP & $>100$ & $\mathrm{AD}$ & $2: 1^{\text {st }} ; 1: 2^{\text {nd }} ; 4: 3^{\text {rd }}$ degree & none & $A P C$ & c. $-19+2475 * 2113+34050 \mathrm{del}$ & whole gene deletion & pathogenic \\
\hline 2 & M-38 & FAP & $>100$ & $\mathrm{AD}$ & $\begin{array}{l}\text { positive family history; } \\
\text { NS }\end{array}$ & G & $A P C$ & c. $-19+5016$ * $* 2113+20168$ del & whole gene deletion & pathogenic \\
\hline 3 & F-29 & FAP & $>100$ & $\mathrm{AD}$ & $2: 1^{\text {st }} ; 1: 2^{\text {nd }}$ degree & none & $A P C$ & c. $-19+5016 \_* 2113+20168 \mathrm{del}$ & whole gene deletion & pathogenic \\
\hline 4 & F-33 & FAP & $>100$ & $\mathrm{AD}$ & $2: 1^{\text {st }} ; 2: 2^{\text {nd }}$ degree & none & $A P C$ & c. $-19+2475 * 2113+34050$ del & whole gene deletion & pathogenic \\
\hline 5 & F-29 & FAP & $>100$ & $\mathrm{AD}$ & $1: 1^{\text {st }} ; 1: 2^{\text {nd }}$ degree & none & $A P C$ & c. $1269 \mathrm{G}>\mathrm{A}$ & p.Trp423Ter & pathogenic \\
\hline 6 & M-35 & FAP & $>100$ & $\mathrm{AD}$ & $1: 1^{\text {st }} ; 5: 2^{\text {nd }}$ degree & none & $A P C$ & c. $1660 \mathrm{C}>\mathrm{T}$ & p.Arg554Ter & pathogenic \\
\hline 7 & M-32 & FAP & $>100$ & $\mathrm{AD}$ & 2: $1^{\text {st }}$ degree & $\mathrm{G}$ & $A P C$ & c.3183_3187del & p.Gln1062Terfs & pathogenic \\
\hline 8 & F-59 & FAP & $>100$ & $\mathrm{AD}$ & $1: 1^{\text {st }} ; 1: 2^{\text {nd }}$ degree & none & $A P C$ & c.3183_3187del & p.Gln1062Terfs & pathogenic \\
\hline 9 & M-38 & FAP & $>100$ & $\mathrm{AD}$ & $1: 1^{\text {st }} ; 1: 2^{\text {nd }}$ degree & none & $A P C$ & c.3199_3202del & p.Ser1068GlyfsTer57 & pathogenic \\
\hline 10 & M-52 & FAP & $>100$ & $\mathrm{AD}$ & no family history & none & $A P C$ & c.3404_3405del & p.Tyr1135fsTer & pathogenic \\
\hline 11 & F-38 & FAP & $>100$ & $\mathrm{AD}$ & $2: 1^{\text {st }} ; 1: 3^{\text {rd }}$ degree & none & $A P C$ & c.3927_3931del & p.Glu1309AspfsTer4 & pathogenic \\
\hline 12 & M-44 & FAP & $>100$ & $\mathrm{AD}$ & 1: $1^{\text {st }}$ degree & none & $A P C$ & c.3927_3931del & p.Glu1309AspfsTer4 & pathogenic \\
\hline 13 & F-39 & FAP & $>100$ & $\mathrm{AD}$ & no family history & none & $A P C$ & c. $904 \mathrm{C}>\mathrm{T}$ & p.Arg302Ter & pathogenic \\
\hline 14 & M-9 & FAP & $>100$ & JP & 1: $1^{\text {st }}$ degree & none & \begin{tabular}{|l} 
BMPRIA; \\
KIT \\
\end{tabular} & $\begin{array}{l}\text { c. }-152-2 \mathrm{~A}>\mathrm{G} ; \\
\text { c. } 2484 \mathrm{C}>\mathrm{T}\end{array}$ & $\begin{array}{l}\text { p.?; } \\
\text { p.Asn828Asn }\end{array}$ & $\begin{array}{l}\text { pathogenic; } \\
\text { VUS } \\
\end{array}$ \\
\hline 15 & M-47 & FAP & $>100$ & $\mathrm{AD}$ & $1: 1^{\text {st }} ; 3: 3^{\text {rd }}$ degree & none & $F L C N$ & c. 1285 dupC & p.His429ProfsTer27 & pathogenic \\
\hline 16 & M-38 & FAP & $>100$ & $\mathrm{AD}$ & no family history & none & unknown & {$[-]$} & {$[-]$} & {$[-]$} \\
\hline 17 & M-39 & oligopolyposis & $\sim 30$ & $\mathrm{AD}$ & 1: $1^{\text {st }}$ degree & none & $A P C$ & c. $256 \mathrm{~A}>\mathrm{T}$ & p.Lys86Ter & pathogenic \\
\hline 18 & M-38 & oligopolyposis & $\sim 10$ & $\mathrm{AD}$ & no family history & none & $A P C$ & c. $3920 \mathrm{~T}>\mathrm{A}$ & p.Ile1307Lys & pathogenic \\
\hline 19 & F-44 & oligopolyposis & $>10$ & $\begin{array}{l}\mathrm{AD} / \\
\mathrm{HP}\end{array}$ & $2: 1^{\text {st }} ; 4: 2^{\text {nd }}$ degree & none & BIMPR1A & c. $1 \mathrm{~A}>\mathrm{G}$ & p.Met1Val & pathogenic \\
\hline 20 & F-40 & oligopolyposis & $\sim 30$ & $\mathrm{AD}$ & no family history & none & MUTYH & c. $734 \mathrm{G}>\mathrm{A} / \mathrm{c} .734 \mathrm{G}>\mathrm{A}$ & $\begin{array}{l}\text { p.Arg245His/ } \\
\text { p.Arg245His }\end{array}$ & pathogenic \\
\hline 21 & M-47 & oligopolyposis & $\sim 10$ & $\mathrm{AD}$ & 2: $1^{\text {st }}$ degree & none & MUTYH & c. $734 \mathrm{G}>\mathrm{A} / \mathrm{c} .734 \mathrm{G}>\mathrm{A}$ & $\begin{array}{l}\text { p.Arg245His/ } \\
\text { p.Arg245His }\end{array}$ & pathogenic \\
\hline 22 & M-48 & oligopolyposis & $>10$ & $\mathrm{AD}$ & 1: $1^{\text {st }}$ degree & none & MUTYH & c. $536 \mathrm{~A}>\mathrm{G} /=$ & p. $\operatorname{Tyr} 179 \mathrm{Cys} /=$ & pathogenic \\
\hline 23 & M-55 & oligopolyposis & $>10$ & $\mathrm{AD}$ & 1: $1^{\text {st }}$ degree & none & MUTYH & c. $536 \mathrm{~A}>\mathrm{G} /=$ & p.Tyr179Cys/= & pathogenic \\
\hline 24 & F-54 & oligopolyposis & $\sim 50$ & $\mathrm{AD}$ & $\begin{array}{l}\text { positive family history; } \\
\text { NS }\end{array}$ & none & $\begin{array}{l}\text { MUTYH; } \\
\text { FANCL }\end{array}$ & $\begin{array}{l}\text { c. } 536 \mathrm{~A}>\mathrm{G} /= \\
\text { c. } 2 \mathrm{~T}>\mathrm{C}\end{array}$ & $\begin{array}{l}\text { p.Tyr179Cys/=; } \\
\text { p.Met1Thr }\end{array}$ & pathogenic \\
\hline 25 & M-67 & oligopolyposis & $50-100$ & $\mathrm{AD}$ & 1: $1^{\text {st }}$ degree & $\mathrm{Pa}$ & NTHL1 & c. $268 \mathrm{C}>\mathrm{T} / \mathrm{c} .806 \mathrm{G}>\mathrm{A}$ & $\begin{array}{l}\text { p.Gln90Ter/ } \\
\text { p.Trp269Ter }\end{array}$ & pathogenic \\
\hline 26 & F-71 & oligopolyposis & 7 & $\mathrm{AD}$ & 2: $1^{\text {st }}$ degree & $\mathrm{E} ; \mathrm{Pa}$ & NTHL1 & c. $268 \mathrm{C}>\mathrm{T} /=$ & p.Gln $90 \mathrm{Ter} /=$ & pathogenic \\
\hline 27 & M-58 & oligopolyposis & $\sim 10$ & $\mathrm{AD}$ & $1: 1^{\text {st }} ; 2: 3^{\text {rd }}$ degree & none & $\begin{array}{l}\text { NTHL1; } \\
\text { RUNX1 } \\
\end{array}$ & $\begin{array}{l}\text { c. } 268 \mathrm{C}>\mathrm{T} /=; \\
\text { c. } 711 \mathrm{G}>\mathrm{C}\end{array}$ & $\begin{array}{l}\text { p.Gln90Ter/=; } \\
\text { p.Gln237His }\end{array}$ & VUS \\
\hline 28 & M-39 & oligopolyposis & $>10$ & $\mathrm{AD}$ & 1: $1^{\text {st }}$ degree & E & $B L M$ & c. $1642 \mathrm{C}>\mathrm{T}$ & p.Gln548Ter & pathogenic \\
\hline 29 & F-53 & oligopolyposis & $>10$ & $\mathrm{AD} / \mathrm{SE}$ & 1: $1^{\text {st }}$ degree & B & CHEK2 & c. 902 delT & p.Leu301TrpfsTer3 & pathogenic \\
\hline 30 & M-53 & oligopolyposis & 21 & $\mathrm{AD}$ & no family history & none & ATM & c. $2149 \mathrm{C}>\mathrm{T}$ & p.Arg717Trp & VUS \\
\hline 31 & M-63 & oligopolyposis & $>10$ & $\mathrm{AD}$ & no family history & none & ATM & c. $9016 \mathrm{G}>\mathrm{C}$ & p.Ala3006Pro & VUS \\
\hline 32 & F-56 & oligopolyposis & NA & $\mathrm{AD}$ & $3: 1^{\text {st }}$ degree & none & MRE11A & c. $1462 \mathrm{C}>\mathrm{T}$ & p.Arg488Cys & VUS \\
\hline 33 & M-45 & oligopolyposis & $\sim 10$ & $\mathrm{AD}$ & no family history & none & MRE11A & c. $1462 \mathrm{C}>\mathrm{T}$ & p.Arg488Cys & VUS \\
\hline 34 & M-46 & oligopolyposis & NA & no data & no family history & none & \begin{tabular}{|l|} 
MRE11A; \\
BLM; \\
DIS3L2 \\
\end{tabular} & $\begin{array}{l}\text { c. } 1462 \mathrm{C}>\mathrm{T} ; \\
\text { c. } 3416 \mathrm{G}>\mathrm{C} ; \\
\text { c. } 1447 \mathrm{C}>\mathrm{G} \\
\end{array}$ & \begin{tabular}{|l|} 
p.Arg488Gly; \\
p.Arg1139Pro; \\
p.Arg483Gly
\end{tabular} & $\begin{array}{l}\text { VUS; } \\
\text { VUS; } \\
\text { VUS }\end{array}$ \\
\hline 35 & M-57 & oligopolyposis & $>10$ & $\mathrm{AD}$ & 1: $1^{\text {st }}$ degree & none & PALB2 & c. $1846 \mathrm{G}>\mathrm{C}$ & p.Asp616His & VUS \\
\hline 36 & F-74 & oligopolyposis & 10 & $\mathrm{AD}$ & 2: $1^{\text {st }}$ degree & none & POLE & c. $2527 \mathrm{~A}>\mathrm{G}$ & p.Ile843Val & VUS \\
\hline 37 & M-38 & oligopolyposis & $\sim 10$ & $\begin{array}{l}\mathrm{AD} / \\
\mathrm{HP}\end{array}$ & $2: 1^{\text {st }} ; 2: 2^{\text {nd }}$ degree & Br; L; P; R & $F H$ & c.1431_1433dupAAA & p.Lys477dup & VUS \\
\hline 38 & M-54 & oligopolyposis & $>30$ & $\mathrm{AD}$ & 1: $1^{\text {st }}$ degree & none & unknown & {$[-]$} & {$[-]$} & {$[-]$} \\
\hline 39 & M-58 & oligopolyposis & $\sim 10$ & $\mathrm{AD}$ & $2: 1^{\text {st }} ; 7: 2^{\text {nd }}$ degree & none & unknown & {$[-]$} & {$[-]$} & {$[-]$} \\
\hline 40 & M-67 & oligopolyposis & $20-30$ & $\mathrm{AD}$ & 2: $1^{\text {st }}$ degree & none & unknown & {$[-]$} & {$[-]$} & {$[-]$} \\
\hline 41 & F-50 & oligopolyposis & $\mathrm{NA}$ & $\mathrm{AD}$ & 1: $1^{\text {st }}$ degree & none & unknown & {$[-]$} & {$[-]$} & {$[-]$} \\
\hline
\end{tabular}

$\mathrm{D}_{\mathrm{X}}$ : diagnosis; ACMG: classification according to the American Collage of Medical Genetics [11]; FAP: familial adenomatous polyposis; NA: not available but <100; VUS: variant of unknown significance; [-]: no variant detected.

a AD: adenomatous; JP: juvenile polyps; HP: hyperplastic; SE: sessile.

b G: gastric cancer; Pa: pancreatic cancer; E: endometrial cancer; B: breast cancer; Br: brain tumor; L: leukemia; P: prostate cancer; R: renal cancer. 
testing even in MSI- negative cases who fulfill the Amsterdam criteria. Half of the detected MMR mutations in the Lynch syndrome patients were located in the MLH1 gene and the other half were equally distributed in $P M S 2$, MSH2 and MSH6 genes. Others have observed a higher frequency of $\mathrm{MSH} 2$ mutations in this syndrome, most probably due to the fact that the recruitment of probands for our study was limited to CRC, and we might have missed some probands with endometrial cancer with this syndrome in which the MSH2 mutations have a higher frequency $[15,16]$. All six patients with MSI+ tumors, in whom we did not detect a pathogenic variant in the MMR genes, had a somatic inactivation of this system, either by promoter methylation of the $M L H 1$ gene (four patients) or by bi-allelic somatic mutation in the $\mathrm{MSH} 2$ gene (two patients). These data indicate that the molecular basis of the hereditary CRC in these families is located in genes associated with other syndromes, which is supported by the fact that two of these patients had pathogenic variants in CHEK2 and FANCL genes.

Deleterious mutations in the $A P C$ gene were present in $13 / 16(80.0 \%)$ patients with classical polyposis, confirming the diagnosis of the FAP syndrome. In the remaining three patients with classical polyposis, we found a known deleterious mutation in the BMPRIA gene in one patient with a hemartomatous component in his polyps, which confirms the diagnosis of juvenile polyposis, a known deleterious mutation in the FLCN gene in one patient, and in one patient we did not detect any pathogenic variants. Deleterious mutations in the FLCN gene were found in patients with Birt-Hogg-Dubé syndrome (BHDS), an autosomal dominant predisposition to multisystem disorders including a higher risk for colorectal neoplasia in c. 1285 insC: p.(His429ProfsTer27) carriers [17-20]. Our index patient with this FLCN mutation exhibited skin fibrofolliculomas characteristic for this syndrome.
We do not yet have complete data on the segregation of the mutation with the FAP phenotype in our family and we cannot exclude the presence of $A P C$ mutation in this patient in the regions not tested by our assay. However, our results further support the notion that certain FLCN gene mutations are involved in colorectal cancerogenesis. The absence of any mutation in the only patient with classical polyposis might be explained by either the presence of a mutation in the non coding/regulatory regions, large rearrangements or mosaicism for a mutation in the $A P C$ gene, or by the presence of a mutation in other gene(s) not analyzed with our approach.

It is worth noting that four mutations, each in the Lynch syndrome and FAP subgroups, were present in $>50.0$ and $>60.0 \%$ of patients, respectively, which allowed for the development of specific assays for initial screening and rationale cascade testing for these syndromes in our population (Staninova-Stojovska et al.; manuscript in preparation).

In the oligopoliposis group, we detected deleterious mutations in $8 / 25(32.0 \%)$ patients. In six of these patients, the variants were present in known highly penetrant genes associated with either autosomal dominant (two in $A P C$ and one in BMPR1A) or recessive (two in MUTYH and one in NTHL1) polyposis syndromes, and two in genes with moderate penetrance (one in CHEK2 and BLM each) [21,22]. In addition, 5/25 (20.0\%) patients were carriers of monoallelic deleterious variants in known genes associated with MUTYH associated polyposis (MAP), and NTHL1 associated polyposis (NAP), autosomal recessive polyposis syndromes (three in MUTYH and two in NTHL1, respectively). Monoallelic carriers of $M U T Y H$ mutations with first degree relatives with CRC are considered at increased risk for the disease and should be offered an early screening program for disease prevention, whereas, as yet, there are no data for risk in monoallelic carriers of NTHL1

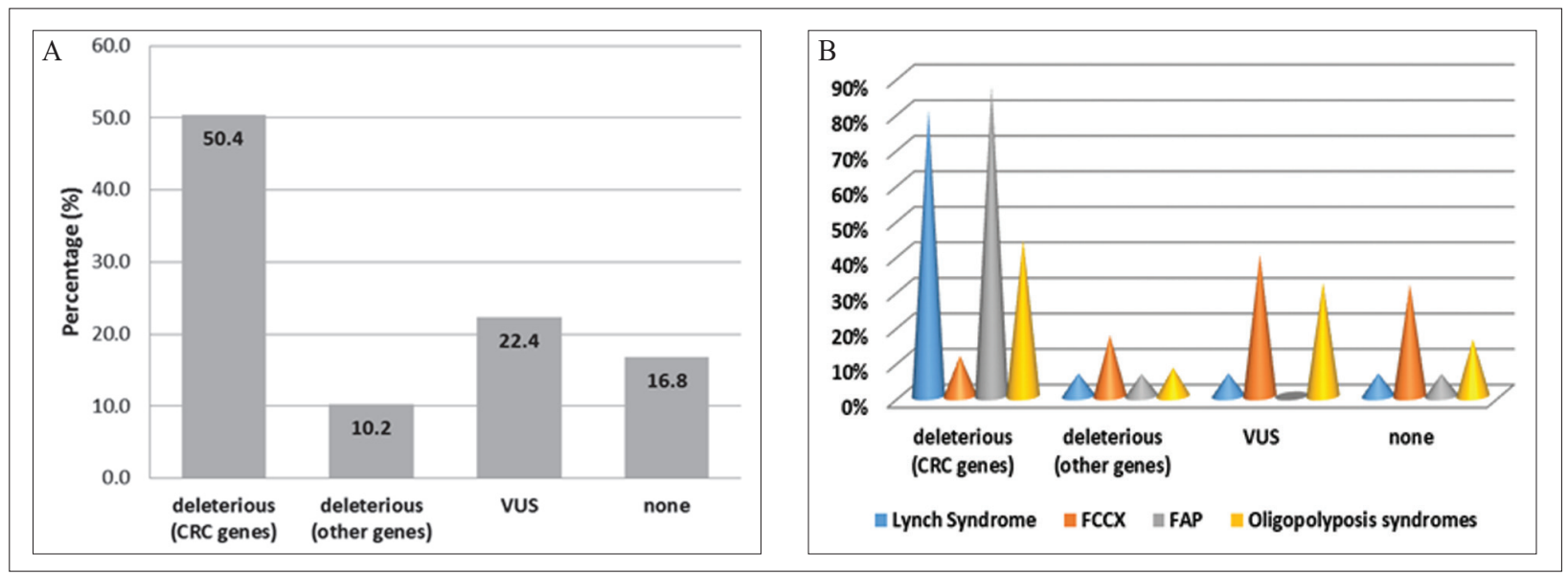

Figure 4. The overall frequency of different types of variants detected in 107 patients with hereditary CRC (A), and their distribution in different clinical subtypes of patients (B). 
pathogenic variants $[22,23]$. In $12 / 25(48 \%)$ patients we did not detect any pathogenic variant, further supporting the notion that the molecular basis of this condition is highly heterogenous and probably involves $\operatorname{defect}(\mathrm{s})$ in other gene(s) not tested in our assay [24].

The lowest frequency of pathogenic variants was present in the group of HNPCC patients with MSS tumors (10/35 or $28.5 \%)$. As mentioned above, four of these patients had Lynch syndrome due to mutations in the MMR genes (two in MSH6 and two in PMS2), which are known to be associated with a lower degree of MSI that might have been undetected by our assay [25-27]. In the other six patients, the variants were present in five DRG, of which four in genes from DNA double-strand break repair pathway (BRIP1 in two patients, BRCA2, FANCM and $C H E K 2$ in one patient each) and one in nucleotide excision repair pathway (ERCC2). Germline mutations in DRG genes have recently been described in individuals with hereditary CRC, though their contribution to a CRC risk is still unknown [14,28-30]. Recent data indicate that DRG defects are also relatively frequent somatic events in CRC, further supporting the notion that the inherited DRG variants are important in CRC carcinogenesis [29,30]. In line with this, some of these variants are described in other hereditary syndromes, predominantly in families with hereditary breast and ovarian cancers (HBOC) as variants with low/moderate penetrance [31]. The observed high prevalence of extracolonic cancers in our families with a DNA damage repair defect is also supporting the premise that mutations in these genes are likely to develop a multitumor phenotype with or without the presence of polyps [32]. The finding of the importance of DRG driven cancerogenesis, particularly in genes involved in homologous recombination, in a significant subset of CRC patients provides a unique opportunity for stratification of patients for platinum-based or target therapy [33-35].

In $42 / 107$ (39.2\%) patients, we did not detect any deleterious variants in the analyzed genes. Nevertheless, 25 different variants of unknown significance were detected in the majority of these patients (24/42 or $57.1 \%)$. Most of these variants had a moderate to low relative risk, as calculated by comparison of their frequency in cancer cases to controls from the Macedonian population or the controls from the GnomAD database (Supplementary Table 2). The VUSs were present predominantly in HNPCC families with MSS tumors (14/42 or 33.3\%) and in families with oligopolyposis $(8 / 42$ or $19.0 \%)$, whereas only two variants were detected in individual patients with the Lynch syndrome [Figure 4(B)]. Most of these variants were present as single variants in individual patients $(20 / 24$ or 83.3\%), whereas three patients with HNPCC with MSS tumors had two different variants and only one patient with oligopolyposys had three different variants (Tables 1 and 2). In addition, only three patients with deleterious mutations also had a VUS. These data strongly suggest that the polygenic inheritance of low/moderate penetrance variants in the 114 analyzed genes is not a major mechanism responsible for the familial CRC type X (FCCX) and oligopolyposis phenotype in our population. It is worth nothing that the majority of these variants, particularly in patients without deleterious mutations, were located in the DRG genes, further supporting their importance in CRC cancerogenesis [Figure 5(B)]. However, additional evidence from functional analysis is needed in order to accurately classify these variants, which might significantly contribute to the current knowledge on the CRC genetic susceptibility.

We could not detect any variants using this approach in 18/107 (16.8\%) patients [Figure 4(A)]. As mentioned above, the majority of these patients (13/18 or $72.2 \%)$ were HNPCC patients (11 with MSS tumors and two with MSI due to somatic inactivation of the MMR genes), 4/18 (22\%) were patients with oligopylopysis and only one patient had a FAP phenotype. Although we cannot exclude the possibility of the presence of a mutation in deep intronic/ regulatory regions of the analyzed genes that could be missed by our approach, we provide further evidence for the genetic heterogeneity of hereditary CRC, particularly in HNPCC families with MSS tumors and in families with oligopolyposis. However, it is interesting to note that endometrial cancer was present in family members of 5/11 HNPCC patients with MSS tumors, which indicates that these are Lynch syndrome families with undetected mutations in MSH6 or PMS2 genes. Further analysis with a whole exome/genome sequencing should clarify whether the increased CRC risk in these families is due to the presence of different high penetrant variants or due to a polygenic risk caused by multiple rare variants in genes not covered

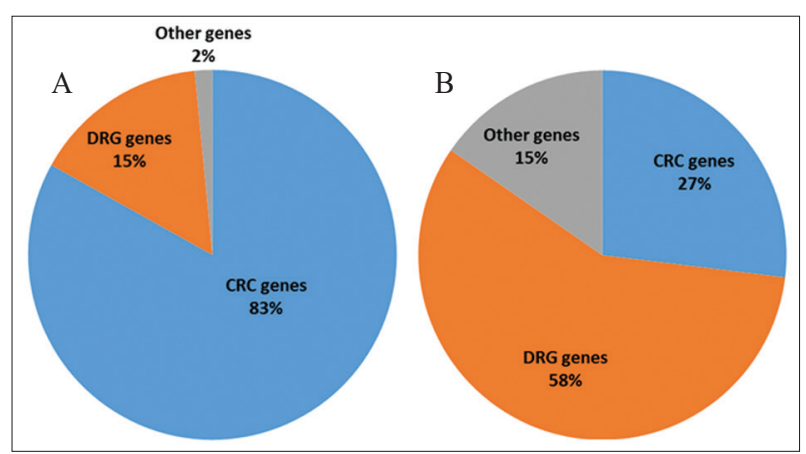

Figure 5. Distribution of deleterious (A) and VUS (B) in known CRC genes (MLH1, MSH2, MSH6, PMS2, APC, MUTYH, NTHL1, BMPR1A, POLE), DRG genes (BRCA2, BLM, CHEK2, BRIP1, PALP2, FANCM, ATM, MRE11, FANCL, ERCC2) and other genes (FLCN, FH, KIT, CDH1, EZH2, CEP57, RUNX1). 
by our assay. The role of gene-environment interactions, as well as epigenetic modifications that are insufficiently explored, should also be considered in understanding the mechanism of the disease development in these families.

\section{ACKNOWLEDGMENTS}

The authors are indebted to Professor Dr. Zoran Kradzov, Professor Dr. Aleksandar Karagjozov, Professor Dr. Nikola Jankulovski, Dr. Toni Josifovski and Dr. Darko Dzambaz from the University Clinic for Abdominal Surgery (Skopje, RN Macedonia) and Professor Dr. Aco Kostovski from the University Clinic for Pediatrics (Skopje, RN Macedonia), for their help in the recruitment of patients, and last but not least, to Professor Dr. Dijana Plaseska-Karanfilska from the Research Center for Genetic Engineering and Biotechnology, Macedonian Academy of Sciences and Arts "Georgi D. Efremov", Skopje, RN Macedonia for providing the unpublished data on the frequencies of certain variants in normal controls from the Macedonian population.

Declaration of Interest. The authors report no conflicts of interest. The authors alone are responsible for the content and writing of this article.

Funding. This study was supported, in part, by a grant CRP/MAC14-01 from the International Centre for Genetic Engineering and Biotechnology (ICGEB), Trieste, Italy, Cooperation inf the Field of Science and Technology Research (Europe) Projects BM1206 and CA17118, grant 07-356/1 from the Macedonian Academy of Sciences and Arts and the funds for science of the Centre for Biomolecuar Farmaceutical Analysis (CBFA), UKIM Faculty of Pharmacy, Skopje, NM Macedonia (all to AJD).

\section{REFERENCES}

1. Lorans M, Dow E, Macrae FA, Winship IM, Buchanan DD. Update on hereditary colorectal cancer: Improving the clinical utility of multigene panel testing. Clin Colorectal Cancer. 2018; 17(2): e293-e305.

2. Loomans-Kropp HA, Umar A. Cancer prevention and screening: The next step in the era of precision medicine. NPJ Precis Oncol. 2019; 3: 3.

3. Boland PM, Yurgelun MB, Boland CR. Recent progress in Lynch syndrome and other familial colorectal cancer syndromes. CA Cancer J Clin. 2018; 68(3): 217-231.

4. Valle L. Genetic predisposition to colorectal cancer: Where we stand and future perspectives. World J Gastroenterol. 2014; 20(29): 9828-9849.
5. Hsu L, Jeon J, Brenner H, Gruber SB, Schoen RE, Berndt SI, et al.; Colorectal Transdisciplinary (CORECT) Study; Genetic and Epidemiology of Colorectal Cancer Consortium (GECCO). A model to determine colorectal cancer risk using common genetic susceptibility loci. Gastroenterology. 2015; 148(7): 1330-1339.e14.

6. Hiljadnikova-Bajro M, Josifovski T, Panovski M, Dimovski AJ. A novel germline MLH1 mutation causing Lynch Syndrome in patients from the Republic of Macedonia. Croat Med J. 2012; 53(5): 496-501.

7. Stefanovska AM, Josifovski T, Panovski M, Jasar D, Zografski G, Efremov GD, et al. Molecular characterization of familial adenomatous polyposis in the Republic of Macedonia. Balkan J Med Genet. 2004; 7(1\&2): 33-40.

8. Zavoral M, Suchanek S, Zavada F, Dusek L, Muzik $\mathrm{J}$, Seifert B, et al. Colorectal cancer screening in Europe. World J Gastroenterol 2009; 15(47): 5907-5915.

9. Pérez-Carbonell L, Alenda C, Payá A, Castillejo A, Barberá VM, Guillén C, et al. Methylation analysis of MLH1 improves the selection of patients for genetic testing in Lynch syndrome. J Mol Diagn. 2010; 12(4): 498-504.

10. Wang K, Li M, Hakonarson H. ANNOVAR: Functional annotation of genetic variants from highthroughput sequencing data. Nucleic Acids Res. 2010; 38(16):e164.

11. Richards S, Aziz N, Bale S, Bick D, Das S, Gastier-Foster J, et al. Standards and guidelines for the interpretation of sequence variants: A joint consensus recommendation of the American College of Medical Genetics and Genomics and the Association for Molecular Pathology. Genet Med. 2015; 17(5): 405-424.

12. Ma H, Difazio S. An efficient method for purification of PCR products for sequencing. BioTechniques. 2008; 44(7): 921-923.

13. Chubb D, Broderick P, Frampton M, Kinnersley B, Sherborne A, Penegar S, et al. Genetic diagnosis of high-penetrance susceptibility for colorectal cancer (CRC) is achievable for a high proportion of familial CRC by exome sequencing. J Clin Oncol. 2015; 33(5): 426-432.

14. Pearlman R, Frankel WL, Swanson B, Zhao W, Yilmaz A, Miller K, et al. Prevalence and spectrum of germline cancer susceptibility gene mutations among patients with early-onset colorectal cancer. JAMA Oncol. 2017; 3(4): 464-471. 
15. Liu B, Parsons RE, Hamilton SR, Petersen GM, Lynch HT, Watson P, et al. hMSH2 Mutations in hereditary nonpolyposis colorectal cancer kindreds. Cancer Res. 1994; 54(17): 4590-4594.

16. Vasen HF, Moslein G, Alonso A, Bernstein I, Bertario L, Blanco I, et al. Guidelines for the clinical management of Lynch syndrome (hereditary non-polyposis cancer). J Med Genet. 2007; 44(6): 353-362.

17. Khoo SK, Giraud S, Kahnoski K, Chen J, Motorna $\mathrm{O}$, Nickolov R, et al. Clinical and genetic studies of Birt-Hogg-Dubé syndrome. J Med Genet. 2002; 39(12): 906-912.

18. Shin JH, Shin YK, Ku JL, Jeong SY, Hong SH, Park $\mathrm{SY}$, et al. Mutations of the Birt-Hogg-Dube (BHD) gene in sporadic colorectal carcinomas and colorectal carcinoma cell lines with microsatellite instability. J Med Genet. 2003; 40(5):364-367.

19. Nahorski MS, Lim DHK, Martin L, Gille JJP, McKay $\mathrm{K}$, Rehal PK, et al. Investigation of the Birt-HoggDubé tumour suppressor gene $(<\mathrm{em}>\mathrm{FLCN}</ \mathrm{em}>)$ in familial and sporadic colorectal cancer. J Med Genet. 2010; 47(6): 385-390.

20. Kashiwada T, Shimizu H, Tamura K, Seyama K, Horie Y, Mizoo A. Birt-Hogg-Dube syndrome and familial adenomatous polyposis: An association or a coincidence? Intern Med. 2012; 51(13): 1789-1792.

21. Stoffel EM, Koeppe E, Everett J, Ulintz P, Kiel M, Osborne J, et al. Germline genetic features of young individuals with colorectal cancer. Gastroenterology. 2018; 154(4): 897-905.e1.

22. Gupta S, Provenzale D, Llor X, Halverson AL, Grady W, Chung DC, et al. NCCN Guidelines insights: Genetic/familial high-risk assessment: Colorectal, Version 2.2019. J Natl Compr Canc Netw. 2019; 17(9): 1032-1041. doi: 10.6004/jncen.2019.0044.

23. Grolleman JE, de Voer RM, Elsayed FA, Nielsen M, Weren RDA, Palles C, et al. Mutational signature analysis reveals NTHL1 deficiency to cause a multitumor phenotype. Cancer Cell. 2019; 35(2): 256-266. e5.(?)

24. Valle L, de Voer RM, Goldberg Y, Sjursen W, Forsti A, Ruiz-Ponte $\mathrm{C}$, et al. Update on genetic predisposition to colorectal cancer and polyposis. Mol Aspects Med. 2019; 69:10-26. doi: 10.1016/j.mam.2019.03.001.

25. Blount J, Prakash A. The changing landscape of Lynch syndrome due to PMS2 mutations. Clin Genet. 2018; 94(1): 61-69.
26. Pino MS, Mino-Kenudson M, Wildemore BM, Ganguly A, Batten J, Sperduti I, et al. Deficient DNA mismatch repair is common in Lynch syndromeassociated colorectal adenomas. J Mol Diagn. 2009; 11(3): 238-247.

27. Berends MJ, Wu Y, Sijmons RH, Mensink RG, van der Sluis T, Hordijk-Hos JM, et al. Molecular and clinical characteristics of MSH6 variants: An analysis of 25 index carriers of a germline variant. Am J Hum Genet. 2002; 70(1): 26-37.

28. Yurgelun MB, Allen B, Kaldate RR, Bowles KR, Judkins T, Kaushik P, et al. Identification of a variety of mutations in cancer predisposition genes in patients with suspected Lynch syndrome. Gastroenterology. 2015; 149(3):604-613.e20.

29. Reilly NM, Novara L, Di Nicolantonio F, Bardelli A. Exploiting DNA repair defects in colorectal cancer. Mol Oncol. 2019; 13(4): 681-700.

30. AlDubayan SH, Giannakis M, Moore ND, Han GC, Reardon B, Hamada T, et al. Inherited DNA-repair defects in colorectal cancer. Am J Hum Genet. 2018; 102(3): 401-414.

31. Nielsen FC, van Overeem Hansen T, Sørensen CS. Hereditary breast and ovarian cancer: New genes in confined pathways. Nat Rev Cancer. 2016; 16(9): 599-612.

32. Knijnenburg TA, Wang L, Zimmermann MT, Chambwe N, Gao GF, Cherniack AD, et al. Genomic and molecular landscape of DNA damage repair deficiency across The Cancer Genome Atlas. Cell Rep. 2019; 23(1): 239-254.e6.

33. Mateo J, Carreira S, Sandhu S, Miranda S, Mossop H, Perez-Lopez R, et al. DNA-repair defects and olaparib in metastatic prostate cancer. N Engl J Med. 2015; 373(18): 1697-1708.

34. Tutt A, Robson M, Garber JE, Domchek SM, Audeh MW, Weitzel JN, et al. Oral poly(ADP-ribose) polymerase inhibitor olaparib in patients with BRCA1 or BRCA2 mutations and advanced breast cancer: A proof-of-concept trial. Lancet. 2010; 376(9737): 235-244.

35. Pishvaian MJ, Blais EM, Brody JR, Rahib L, Lyons $\mathrm{E}$, Arbeloa PD, et al. Outcomes in patients with pancreatic adenocarcinoma with genetic mutations in DNA damage response pathways: Results from the Know Your Tumor Program. JCO Precision Oncology. 2019; 37:(4 Suppl): 191. 